\title{
Investigación experimental sobre los modelos normativos de fisuración en piezas de hormigón armado sometidas a flexión pura
}

\author{
Experimental research on standard cracking patterns in \\ reinforced concrete members subjected to pure bending
}

E. Calderón $^{(*)}$, J. Fernández $z^{(*)}$

RESUMEN

La presente investigación analiza la idoneidad de las formulaciones propuestas por EHE, Eurocódigo 2 y ACl 318 Building Code para el control de la fisuración en elementos de hormigón armado mediante el ensayo de elementos a escala real, 14 vigas a flexión pura. Además analiza la influencia de la variación de parámetros tales como el diámetro, cuantía y disposición de armadura comprimida. En esta investigación se analiza el ajuste de las formulaciones teóricas antes referidas y los resultados experimentales obtenidos, con resultados en algunos casos más desfavorables que los previstos. También pone de manifiesto la notable influencia de algunos parámetros, tales como la cuantía en el control de la fisuración, y por el contrario, la poca influencia de parámetros tales como el diámetro, la separación de armaduras, y la disposición de armadura comprimida. De acuerdo con los resultados obtenidos en la investigación, finalmente se evalúan posibles variantes de la formulación existente, concretamente de la formulación que ha presentado una tendencia más adecuada frente a los resultados de los ensayos (EC-2), con objeto de mejorar el ajuste de dicha formulación a los resultados experimentales.

\section{SUMMARY}

The present study analyzes the suitability of the formulas proposed in EHE, Eurocode 2 and $\mathrm{ACI}$ Building Code 318 for crack control in reinforced concrete by testing 14 full-scale beams subjected to pure bending. It also explores the effect of varying parameters such as diameter, steel ratio and compression reinforcement arrangement and analyzes the fit between the above theoretical procedures and the experimental findings (less favourable than expected in some cases). The article also discusses the considerable impact of certain parameters, such as steel ratio, on crack control, and the scant effect of parameters such as diameter, reinforcement spacing and the arrangement of compression reinforcement. Pursuant to the results obtained in this study, possible variations on existing formulas are evaluated, specifically on the formula exhibiting a tendency that came closest to reflecting the test results (EC-2), with a view to improving the fit between that formula and the experimental results.

Keywords: crack control; crack spacing; crack width; pure bending; reinforcing bar diameter; mechanical reinforcement ratio.
Palabras clave: control fisuración; separación de fisuras; abertura de fisuras; flexión pura; diámetro de armadura; cuantía mecánica.
$451-14$

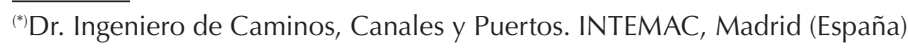

${ }^{(* *)}$ Dr. Ingeniero de Caminos, Canales y Puertos (UPM), Madrid (España)

Persona de contacto/Corresponding author: ecalderon@intemac.es
}

Fecha de recepción: 02-07-09 Fecha de aceptación: 16-09-09 


\section{INTRODUCCIÓN}

El diseño de las estructuras de hormigón armado exige garantizar tanto unas adecuadas condiciones de seguridad como unas apropiadas condiciones de servicio. El uso de la estructura puede verse afectado por una fisuración excesiva, deformaciones excesivas, oscilaciones perturbadoras, reducida capacidad frente a fuego, corrosión del acero, etc. Aunque disponemos de muchas herramientas para impedir que se produzcan estos fenómenos, algunos de ellos son inevitables, y lo más que podemos hacer es controlarlos y mantenerlos en límites aceptables.

Es prácticamente imposible evitar que aparezcan fisuras en elementos de hormigón armado, dada la reducida resistencia a tracción del hormigón, aún para tensiones de tracción pequeñas, ya sea por la actuación de cargas exteriores o por esfuerzos inducidos. Sin embargo, pese a que se trata de un proceso casi inevitable, debemos diseñar las estructuras limitando la posible abertura máxima de éstas por razones de durabilidad (1-5), estanqueidad, estética (6) y para reducir el efecto psicológico (7) que produciría sobre los usuarios.

A lo largo de la historia del hormigón armado son muchos los autores que han dedicado su investigación a la fisuración, Saliger (1936), Lossier (1936), Rüsch (1957), Rehm (1957), Watstein (1959), Mathey (1959), Broms (8) (1965), Lutz (9) (1965), Borges (10) (1966), Beeby (11-12) (1966), Nawy (13) (1968), Frosch (14-15) (1999), entre otros. Los estudios realizados sobre la fisuración en elementos de hormigón armado han tratado de establecer unos valores máximos admisibles para la abertura de fisuras en función del tipo de exposición de la estructura, así como de establecer fórmulas que proporcionen la abertura previsible de fisura en función de las características de la pieza y de su estado tensional.

La influencia de diversos parámetros en la fisuración y la complejidad de un buen ajuste entre los estudios teóricos y los ensayos realizados plantean la necesidad de seguir analizando la fisuración en elementos de hormigón armado, buscando la formulación que mejor se ajuste a este fenómeno. Entre los parámetros que intervienen en las diversas fórmulas planteadas por las distintas normativas internacionales para la estimación de la abertura de fisuras en elementos de hormigón armado, se encuentra el recubrimiento de las armaduras, el diámetro empleado, su separación, la cuantía eficaz, y la tensión del acero. La finalidad de esta investigación es comparar y analizar los resultados de separación y abertura de fisuras obtenidas en una campa- ña experimental con los valores deducidos según las formulaciones propuestas por EHE (16), Eurocódigo 2 (17) y ACl 318 Building Code (18). Dichas normativas abordan el fenómeno de la fisuración por dos vías muy diferentes, como se indica más adelante. Específicamente, se ha analizado la influencia de la variación de los siguientes parámetros sobre la fisuración en elementos sometidos a flexión pura: cuantía de armadura dispuesta, diámetro de las armaduras y disposición de armadura comprimida.

\section{OBJETO DE LA INVESTIGACIÓN}

El objetivo de la investigación es verificar la idoneidad de las formulaciones propuestas por las últimas versiones de EHE, ACl 318 Building Code y Eurocódigo 2 para el control de la fisuración en elementos de hormigón armado a escala real, mediante el ensayo de 14 vigas a flexión pura. Además se analiza la influencia de la variación de parámetros como el diámetro, cuantía y disposición de armadura comprimida en la adecuación de los resultados experimentales y los teóricos. Esta investigación demuestra la falta de ajuste de las formulaciones teóricas antes referidas y los resultados experimentales obtenidos, con resultados reales en general más desfavorables que los previstos.

\section{TRATAMIENTO NORMATIVO DE LA FISURACIÓN}

A continuación se expone, a modo de resumen, el diferente tratamiento dado por la normativa española EHE y las dos normativas internacionales consideradas, ACI 318 Building Code y Eurocódigo 2, para el control de la fisuración en elementos de hormigón armado.

Previamente indicamos el significado de los parámetros comunes empleados por las tres formulaciones expuestas a continuación:

$\mathrm{w}=$ abertura de fisura.

$\mathrm{w}_{\mathrm{k}}=$ abertura característica de fisura.

$\mathrm{w}_{\max }=$ abertura máxima de fisura admitida según la clase de exposición de la estructura. $\mathrm{s}=$ separación de fisuras.

$\mathrm{s}_{\mathrm{m}}=$ separación media de fisuras.

$\mathrm{c}=$ recubrimiento geométrico de la armadura.

$\mathrm{d}_{\mathrm{c}}=$ recubrimiento mecánico de la armadura.

$d_{\phi}=$ distancia entre barras longitudinales traccionadas.

$\phi=$ diámetro de la barra traccionada más gruesa o diámetro equivalente en el caso de grupo de barras.

$\mathrm{A}_{\mathrm{c}, \text { eficaz }}=$ área de hormigón de zona de recu- 
brimiento, en donde las barras a tracción influyen de forma efectiva en la abertura de las fisuras.

$\mathrm{A}_{\mathrm{s}}=$ sección total de las armaduras situadas en el área $A_{c, e f i c a z}$.

$\rho_{\mathrm{p}, \text { eff }}=$ cuantía geométrica de armadura efectiva, $A_{s} / A_{\text {ceffi }}$.

$\mathrm{f}_{\mathrm{ctm}}=$ es el valor medio de la resistencia del hormigón a tracción a la edad $t$ a la que aparece la fisura.

$\mathrm{E}_{\mathrm{cm}}=$ módulo de deformación media del hormigón.

$\mathrm{E}_{\mathrm{s}}=$ módulo de deformación longitudinal del acero.

$\sigma_{\mathrm{s}}=$ tensión en servicio de la armadura pasiva traccionada en la hipótesis de sección fisurada.

$\sigma_{\mathrm{sr}}=$ tensión de la armadura traccionada en la sección fisurada en el instante en que se fisura el hormigón, lo cual se supone que ocurre cuando la tensión de tracción en la fibra más traccionada de hormigón alcanza la resistencia media a tracción del hormigón.

$\varepsilon_{\mathrm{sm}}=$ deformación media del acero teniendo en cuenta la colaboración del hormigón entre fisuras.

$\varepsilon_{\mathrm{cm}}=$ es la deformación media del hormigón.

\subsection{Control de la fisuración según EHE}

La comprobación general del Estado Límite de Fisuración según la Instrucción EHE, consiste en satisfacer la siguiente inecuación:

$\mathrm{w}_{\mathrm{k}} \leq \mathrm{w}_{\max }$

siendo,

$\mathrm{w}_{\mathrm{k}}=\beta \cdot \mathrm{s}_{\mathrm{m}} \cdot \varepsilon_{s m}$

$\beta=$ coeficiente que relaciona la abertura media de fisura con el valor característico, y vale 1.3 para fisuración producida por acciones indirectas solamente y 1.7 para el resto de casos.

$\mathrm{s}_{\mathrm{m}}=2 \cdot c+0.2 \cdot \mathrm{d}_{\phi}+0.4 \cdot k_{1} \cdot \phi \cdot \frac{A_{c, e f i c a z}}{A_{s}}$

$\varepsilon_{\mathrm{sm}}=\frac{\sigma_{s}}{E_{s}} \cdot\left(1-k_{2} \cdot\left(\frac{\sigma_{s r}}{\sigma_{s}}\right)^{2}\right) \nless 0.4 \cdot \frac{\sigma_{s}}{E_{s}}$

$\mathrm{k}_{1}$ = coeficiente que representa la influencia del diagrama de tracciones en la sección.

$\mathrm{k}_{2}$ = coeficiente de valor 1.0 para los casos de carga instantánea no repetida y 0.5 para los restantes.

\subsection{Control de la fisuración según Eu- rocódigo 2}

El Eurocódigo 2 establece el cálculo de la armadura mínima necesaria para controlar los fenómenos de fisuración, y la comprobación de la abertura máxima de fisura de acuerdo con la siguiente expresión:

$\mathrm{W}_{\mathrm{k}} \leq \mathrm{W}_{\max }$

donde,

$\mathrm{w}_{\mathrm{k}}=\mathrm{s}_{\mathrm{rmax}} \cdot \varepsilon_{\mathrm{m}}$

$\mathrm{s}_{\mathrm{r}, \max }=$ separación máxima entre fisuras que resulta de la expresión (23):

$\mathrm{s}_{\mathrm{r} \text { max }}=\mathrm{k}_{3} \cdot \mathrm{c}+\mathrm{k}_{1} \cdot \mathrm{k}_{2} \cdot \mathrm{k}_{4} \cdot\left(\phi / \rho_{\mathrm{p} \mathrm{eff}}\right)$

Cabe señalar que dicha expresión ya incluye un coeficiente de corrección para obtener directamente la separación máxima (característica) de fisuras y no la media. Dicho coeficiente es 1.7.

$\varepsilon_{\mathrm{m}}=\varepsilon_{\mathrm{sm}}-\varepsilon_{\mathrm{cm}}=\frac{\sigma_{\mathrm{s}}-k_{t} \cdot \frac{\mathrm{f}_{\mathrm{ctm}}}{\rho_{\mathrm{peff}}} \cdot\left(1+\alpha_{e} \cdot \rho_{\mathrm{peff}}\right)}{E_{s}} \geq 0.6 \cdot \frac{\sigma_{\mathrm{s}}}{E_{s}}$

$\mathrm{k}_{1}=$ coeficiente que considera las propiedades de adherencia de las barras, siendo 0.8 en el caso de barras corrugadas y de 1.6 para el de barras lisas.

$k_{2}$ = coeficiente que tiene en cuenta la forma de distribución de deformaciones, siendo de 0.5 para flexión, y de 1.0 para tracción pura.

En el caso de tracción excéntrica o para áreas locales, se utilizarán valores intermedios de $k_{2}$.

$\mathrm{k}_{3}, \mathrm{k}_{4}=$ coeficientes cuyo valor viene recomendado en el Anejo Nacional de cada país. $k_{t}$ = factor que depende de la duración de la carga, siendo de 0.6 para cargas instantáneas o de corta duración, y de 0.4 para cargas mantenidas o repetidas.

$\alpha_{\mathrm{e}}=$ relación $\mathrm{E}_{\mathrm{s}} / \mathrm{E}_{\mathrm{cm}}$.

El Eurocódigo incluye un método simplificado para el control de la fisuración en losas sometidas a flexión (con esfuerzos de tracción despreciables), limitando el diámetro de las barras y la separación de éstas en función de la tensión del acero.

\subsection{Control de la fisuración según $\mathrm{ACI} 318$ Building Code}

La normativa norteamericana $\mathrm{ACl}$ Building Code sufrió un cambio sustancial en la edición del año 1999 respecto a la anterior (1995) en cuanto al tratamiento de la fisuración (19-21). Mediante el análisis de las investigaciones Ilevadas a cabo por Gergely y Lutz (9), Beeby (11-12), y Frosch (14), y las formulaciones propuestas por éstos para el 
control de la abertura de fisuras, se estableció una nueva ecuación para el cálculo directo de la separación máxima de las armaduras. Fueron fundamentalmente las investigaciones llevadas a cabo en la década de los noventa por Frosch, las que produjeron un cambio sustancial en la formulación recogida en la normativa norteamericana a partir de 1999. En dichas investigaciones, apoyadas entre otras por las realizadas por Broms, se establecía la dependencia que existía entre la separación de fisuras, y el recubrimiento y la separación de las armaduras mediante:

$w=\psi_{s} \cdot \frac{\sigma_{s}}{E_{s}} \cdot \beta \cdot \sqrt{d_{c}{ }^{2}+\left(d_{\phi} / 2\right)^{2}}$

donde,

$\Psi_{\mathrm{s}}=$ factor de separación de fisuras: 1.0 para obtener la separación mínima de fisuras; 1.5 para obtener la media; y 2.0 para obtener la máxima.

$\beta=1+0.08 \cdot d_{c}$

De dicha expresión se deduce:

$d_{\phi}=2 \cdot \sqrt{\left(\frac{w \cdot E_{s}}{\psi_{s} \cdot \sigma_{s} \cdot \beta}\right)^{2}-d_{c}{ }^{2}}$

Basándose en dicha expresión Frosch propone la siguiente ecuación simplificada para limitar la separación entre armaduras longitudinales, como se muestra en la Fig. 1.

$d_{\phi}=12 \cdot \alpha_{s} \cdot\left(2-\frac{d_{c}}{3 \cdot \alpha_{s}}\right) \leq d_{\phi}=12 \cdot \alpha_{s}$

$\alpha_{s}=\frac{36}{\sigma_{s}}$

Esta ecuación simplificada sirvió de base para establecer la ecuación definitiva recogida en la normativa norteamericana. La ecuación que se incluye en el ACl 318-99 (21) es la siguiente:

$d_{\phi}=\frac{540}{\sigma_{s}}-2.5 \cdot d_{c} \leq 12 \cdot \frac{36}{\sigma_{s}}$

donde $\sigma_{\mathrm{s}}$ se puede tomar de forma aproximada como $0.60 \cdot f_{\mathrm{yk}}$ (tensión de límite elástico del acero).

El ACl 318-06 (22) modifica ligeramente la expresión anterior (incluida también en la edición de 2005), resultando:

$d_{\phi}=\frac{600}{\sigma_{s}}-2.5 \cdot d_{c} \leq 12 \cdot \frac{40}{\sigma_{s}}$

Dicha expresión se mantiene en la normativa ACl 318-08 (18) actualmente en vigor.

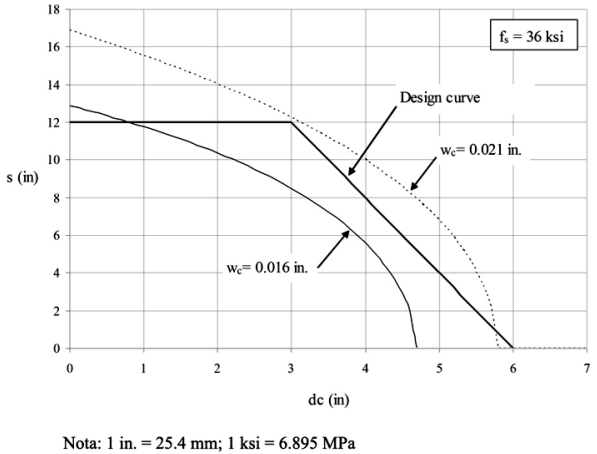

\section{INVESTIGACIÓN EXPERIMENTAL REALIZADA}

Para el análisis de la adecuación de los resultados experimentales y los teóricos las variables investigadas han sido las siguientes:

- Cuantía mecánica de armadura traccionada: Tres cuantías, alta (entre 0.45 y 0.61), media (del orden de 0.22) y baja (del orden de 0.09).

- Cuantía mecánica de armadura comprimida: Para las vigas 1 a 8 se dispuso sólo armadura de montaje (2ф8), y para las vigas 9 a 11, y 12 a 14, cuantías media (0.14) y alta (0.29), respectivamente.

-Diámetro de las armaduras traccionadas: Tres diámetros, finos $(\phi 10)$, medios $(\phi 16)$ y gruesos $(\phi 20)$.

Teniendo en cuenta dichas variables se han ensayado 14 vigas de hormigón armado a flexión pura para obtener un esquema de fisuración lo más uniforme posible. Así, los esfuerzos de flexión son constantes a lo largo de toda la zona instrumentada, y no están influenciados por tensiones de corte que distorsionarían el esquema de fisuración previsto.

Las vigas han sido construidas y ensayadas en la losa de ensayos del Laboratorio Central del Instituto Técnico de Materiales y Construcciones (INTEMAC) sito en Madrid (España).

A continuación se indican los materiales empleados, la definición de las vigas ensayadas y la metodología de ensayo.

\subsection{Materiales}

Las vigas se fabricaron con un hormigón, representativo de los utilizables en elementos estructurales de edificación, elaborado con un tamaño máximo de árido de 20 mm y consistencia blanda. Se utilizó una dosificación representativa de un hormigón de $25 \mathrm{MPa}$ de resistencia característica a la edad de ensayo de las vigas. Las armaduras de las vigas se fabricaron con acero de dureza natural B400S, 
correspondiente a un acero de límite elástico de 400 MPa. Para obtener los parámetros mecánicos del hormigón vertido en cada una de las vigas, se fabricaran probetas cilíndricas moldeadas de hormigón y probetas prismáticas normalizadas, efectuándose los ensayos de caracterización siguientes:

\section{Ensayos de compresión}

Sobre ocho probetas cilíndricas. Cuatro de ellas ensayadas a la misma edad de ensayo de las vigas, dos curadas en idénticas condiciones que el hormigón de las mismas (ambiente de laboratorio) y las otras dos en cámara estándar. Las cuatro probetas restantes se ensayaron a los 28 días de edad, dos curadas en ambiente de laboratorio y dos curadas en cámara.

\section{Ensayos de tracción indirecta}

Sobre seis probetas cilíndricas. Dos de ellas ensayadas a la misma edad de ensayo de las vigas, curadas en idénticas condiciones que el hormigón de las mismas (ambiente de laboratorio). Las cuatro probetas restantes se ensayaron a los 28 días de edad, dos curadas en ambiente de laboratorio y dos curadas en cámara.

\section{Ensayos de flexotracción}

Para determinar el momento de fisuración de las vigas se ensayaron seis probetas prismáticas, distribuidas análogamente a como se ha indicado para los ensayos de tracción indirecta.

Ensayos para la determinación del módulo de deformación

Se efectuaron sobre dos probetas cilíndricas a la edad de ensayo de las vigas y curadas en ambiente de laboratorio.

En lo que hace referencia al acero, se realizaron ensayos sobre muestras representativas de cada una de las capas de las armaduras longitudinales de las vigas para efectuar ensayos de tracción, con determinación de las características geométricas y mecánicas, y ensayos de doblado simple, doblado-desdoblado de las barras y geometría del corrugado del diámetro utilizado. Las características mecánicas de los materiales, hormigón y acero, para cada una de las vigas ensayadas se muestran en las Tablas 1 y 2 , respectivamente.

\subsection{Descripción de las vigas ensayadas}

Se fabricaron 14 vigas rectangulares de 250 $\mathrm{mm}$ de ancho por $500 \mathrm{~mm}$ de canto de 6.50 $\mathrm{m}$ de longitud total, y biapoyadas a $1.25 \mathrm{~m}$
Tabla 1

Características del hormigón empleado en los ensayos (MPa)*+

\begin{tabular}{|c|c|c|c|c|}
\hline Viga & $\begin{array}{c}\text { Resistencia a } \\
\text { compresión }\end{array}$ & $\begin{array}{c}\text { Módulo de } \\
\text { deformación }\end{array}$ & $\begin{array}{c}\text { Resistencia a } \\
\text { tracción } \\
\text { indirecta }\end{array}$ & $\begin{array}{c}\text { Resistencia a } \\
\text { flexotracción }\end{array}$ \\
\hline 1 & 24.06 & 21092 & 2.63 & 3.38 \\
\hline 2 & 22.59 & 20689 & 2.71 & 3.39 \\
\hline 3 & 24.38 & 20753 & 2.72 & 3.78 \\
\hline 4 & 26.14 & 21484 & 2.84 & 3.45 \\
\hline 5 & 24.40 & 20312 & 2.22 & 4.54 \\
\hline 6 & 24.62 & 20248 & 2.43 & 4.00 \\
\hline 7 & 21.90 & 20297 & 2.74 & 3.36 \\
\hline 8 & 24.11 & 22269 & 2.96 & 3.46 \\
\hline 9 & 26.00 & 23294 & 2.98 & 3.66 \\
\hline 10 & 28.18 & 21611 & 2.79 & 3.65 \\
\hline 11 & 24.18 & 19914 & 3.09 & 3.52 \\
\hline 12 & 27.08 & 23500 & 3.34 & 3.85 \\
\hline 13 & 26.27 & 24138 & 3.12 & 3.58 \\
\hline 14 & 24.13 & 20758 & 3.54 & 2.59 \\
\hline
\end{tabular}

* Las probetas moldeadas han sido ensayadas bajo las mismas condiciones de curado y con la misma edad que las vigas.

+ Valores medios.

Tabla 2

Características del acero empleado en los ensayos (MPa)*

\begin{tabular}{|c|c|c|c|c|}
\hline Viga & Límite elástico & $\begin{array}{c}\text { Resistencia a } \\
\text { tracción }\end{array}$ & $\begin{array}{c}\text { Deformación de } \\
\text { rotura }\end{array}$ & $\begin{array}{c}\text { Módulo de } \\
\text { deformación }\end{array}$ \\
\hline 1 & 514.58 & 620.53 & 12.8 & 204048 \\
\hline 2 & 492.81 & 594.29 & 14.5 & 203558 \\
\hline 3 & 521.48 & 514.85 & 14.0 & 203312 \\
\hline 4 & 502.62 & 608.51 & 14.5 & 202577 \\
\hline 5 & 502.57 & 600.13 & 15.8 & 202086 \\
\hline 6 & 515.22 & 603.27 & 11.4 & 202577 \\
\hline 7 & 502.57 & 598.16 & 13.5 & 205029 \\
\hline 8 & 513.36 & 605.13 & 14.1 & 203558 \\
\hline 9 & 504.63 & 608.56 & 14.6 & 204048 \\
\hline 10 & 510.27 & 604.35 & 13.4 & 201596 \\
\hline 11 & 510.87 & 603.04 & 12.3 & 204440 \\
\hline 12 & 508.60 & 612.54 & 14.6 & 205029 \\
\hline 13 & 491.19 & 597.53 & 14.7 & 202413 \\
\hline 14 & 517.61 & 604.81 & 13.9 & 202478 \\
\hline
\end{tabular}

Valores medios.

de sus extremos. En la Tabla 3 se indican los esquemas de armado de cada una de las vigas 2. Secciones transversales de algunas de las vigas ensayadas. ensayadas y en la Fig. 2, a modo de ejemplo, los croquis de sección de algunas de ellas. Las vigas 1 a 3 presentan una cuantía mecánica (ळ) alta, entre 0.45 y 0.48 , cubierta con diámetros gruesos ( $\phi 25 \mathrm{~mm})$, medios $(\phi 16 \mathrm{~mm})$, y finos $(\phi 10 \mathrm{~mm})$. Las vigas 4 a 6 presentan una $\varpi$ media, entre 0.22 y 0.23 , cubierta con los tres tipos de diámetros antes citados. Las vigas 7 y 8 presentan una $\vee$ baja, de 0.09 , cubierta con diámetros medios $(\phi 16 \mathrm{~mm})$ y finos $(\phi 10 \mathrm{~mm})$, respectivamente. Por último,

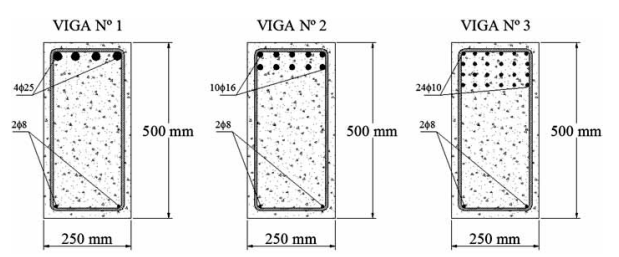


3. Croquis de disposición del ensayo.

4. Vista de la disposición del ensayo. Laboratorio Central de INTEMAC.
Tabla 3

Esquemas de armado de las vigas ensayadas

\begin{tabular}{|c|c|c|}
\hline Viga & $\begin{array}{c}\text { Armadura } \\
\text { traccionada }\end{array}$ & $\begin{array}{c}\text { Armadura } \\
\text { comprimida }\end{array}$ \\
\hline 1 & $4 \phi 25$ & $2 \phi 8$ \\
\hline 2 & $10 \phi 16$ & $2 \phi 8$ \\
\hline 3 & $24 \phi 10$ & $2 \phi 8$ \\
\hline 4 & $2 \phi 25$ & $2 \phi 8$ \\
\hline 5 & $5 \phi 16$ & $2 \phi 8$ \\
\hline 6 & $12 \phi 10$ & $2 \phi 8$ \\
\hline 7 & $2 \phi 16$ & $2 \phi 8$ \\
\hline 8 & $5 \phi 10$ & $2 \phi 8$ \\
\hline 9 & $5 \phi 25$ & $3 \phi 16$ \\
\hline 10 & $12 \phi 16$ & $3 \phi 16$ \\
\hline 11 & $30 \phi 10$ & $3 \phi 16$ \\
\hline 12 & $5 \phi 25$ & $4 \phi 20$ \\
\hline 13 & $12 \phi 16$ & $4 \phi 20$ \\
\hline 14 & $30 \phi 10$ & $4 \phi 20$ \\
\hline
\end{tabular}
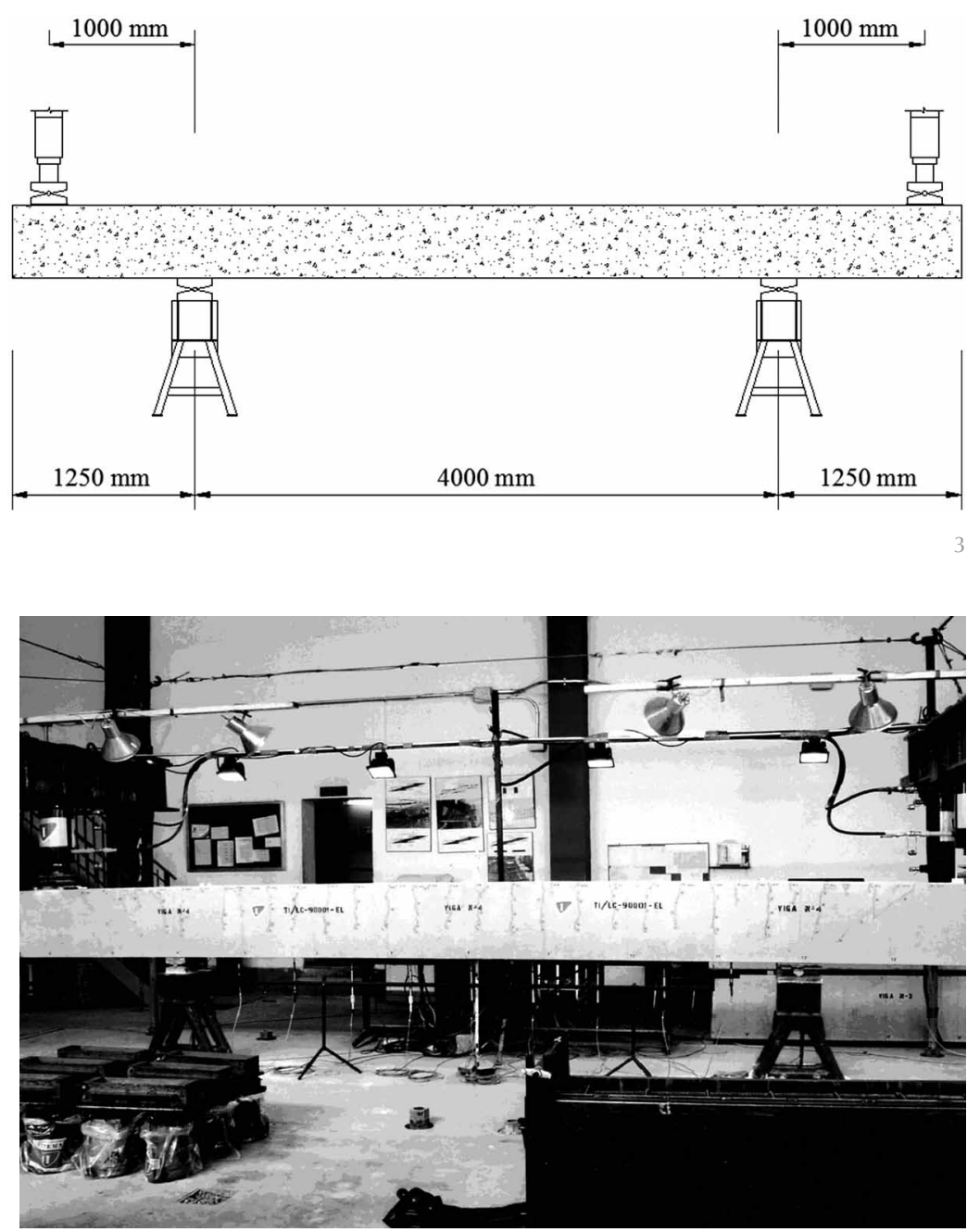

las vigas 9 a 11 y 12 a 14 presentan una $v$ alta, entre 0.59 y 0.61 , cubierta para los tres tipos de diámetros y para dos tipos de cuantía mecánica de armadura comprimida $\left(\varpi^{\prime}\right)$, de 0.14 y de 0.29 , respectivamente.
El esquema del ensayo se muestra en la Fig. 3. La carga se aplicaba en los voladizos a $1 \mathrm{~m}$ del apoyo, obteniéndose entonces momento flector constante y esfuerzo cortante nulo en los $4 \mathrm{~m}$ de elemento entre apoyos. En la Fig. 4 se muestra una fotografía de una de las vigas ensayadas.

Las piezas fueron ensayadas hasta rotura pasando por distintos escalones. En primer lugar se fijó el escalón de carga correspondiente al momento flector resistente teórico de cada una de las vigas (carga de cálculo). Fijado este escalón fue posible obtener directamente la carga de ensayo para el escalón de servicio dividiendo la carga de calculo entre un coeficiente aproximado de mayoración de acciones de 1.6. Fijados estos dos escalones, correspondientes a la carga de servicio y la carga de cálculo se distribuyeron el resto de escalones uniformemente (aproximadamente), incluyendo entre los resultantes el escalón de fisuración.

Se han registrado los esquemas de fisuración obtenidos para cada una de las vigas, anotando la separación de fisuras y las aberturas de cada una de las fisuras registradas para cada uno de los escalones de carga a nivel de la capa de armadura traccionada más exterior. Para la medida de la abertura de fisuras se emplearon fisurómetros de transparencia y lupa graduada con apreciación de $0.05 \mathrm{~mm}$.

\section{ANÁLISIS DE LOS RESULTADOS EXPERIMENTALES OBTENIDOS}

Mediante el tratamiento de las medidas registradas sobre la abertura y separación de fisuras se obtiene el valor de abertura media de fisuras para la carga de servicio de cada viga ensayada, y la separación media de fisuras para cada una de las vigas. A continuación se exponen y comentan los resultados experimentales obtenidos y los resultados deducidos según las tres normativas antes referidas (EHE, EC-2 y ACl). También se establece una comparativa entre los resultados experimentales y los resultados teóricos para valorar el grado de adecuación de las normativas consideradas a la campaña experimental.

\section{Abertura de fisura}

En la Fig. 5 se muestran los valores de abertura media de fisuras para el escalón de servicio obtenidos en los ensayos realizados.

Para cuantías altas (vigas 1 a 3 ) se obtienen valores comprendidos entre $0.08 \mathrm{~mm}$ y 0.11 $\mathrm{mm}$, para cuantías medias (vigas 4 a 6) se obtienen valores entre $0.14 \mathrm{~mm}$ y $0.19 \mathrm{~mm}$, y para cuantías bajas (vigas 7 y 8) se obtienen valores entre $0.14 \mathrm{~mm}$ y $0.32 \mathrm{~mm}$. Cabe seña- 


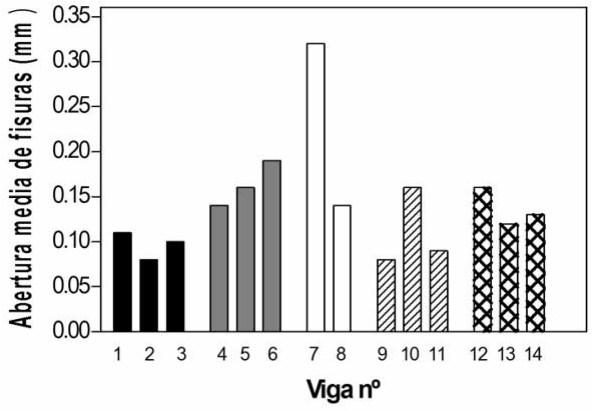

Cuantía de armadura traccionada alta Cuantía de armadura traccionada media Cuantía de armadura traccionada baja Cuantía de armadura traccionada muy alta y cuantía de armadura comprimida media xx Cuantía de armadura traccionada muy alta y cuantía de armadura comprimida alta

lar que en el caso de la viga 7, dada la baja cuantía dispuesta, presentaba tras su fabricación fisuras debidas a la contracción térmica inicial del hormigón (24). Las aberturas de dichas fisuras fueron medidas y descontadas de las obtenidas durante el ensayo.

Se deduce que según aumenta la cuantía mejora el control de fisuración y se reduce la dispersión entre los resultados obtenidos para una misma cuantía. No se observa una reducción de la abertura de fisura al reducir el diámetro de las armaduras con una misma cuantía de armadura. Conclusiones análogas fueron obtenidas, entre otros, por Beeby (23) en sus estudios realizados sobre la influencia del parámetro $\phi / \rho_{\text {eff }}$.

En cuanto a las vigas de cuantía elevada, con y sin armadura comprimida, no se observa influencia alguna en la disposición de la misma. Los resultados obtenidos están comprendidos entre $0.08 \mathrm{~mm}$ y $0.16 \mathrm{~mm}$. Además, se observa la variabilidad de resultados que puede haber entre vigas con idéntica disposición de armadura traccionada.

En la Fig. 6 se muestran los valores de abertura media de fisuras para el escalón de servicio obtenidos de acuerdo con la formulación propuesta por EHE. Esta formulación tiene en cuenta los parámetros que se analizan en la presente investigación, si bien no considera la influencia de la disposición de armadura comprimida.

Para cuantías altas (incluyendo las vigas con y sin armadura comprimida) se obtienen valores comprendidos entre $0.11 \mathrm{~mm}$ y 0.15 $\mathrm{mm}$, para cuantías medias se obtienen valores entre $0.13 \mathrm{~mm}$ y $0.21 \mathrm{~mm}$, y para cuantías bajas se obtienen valores entre $0.07 \mathrm{~mm}$ y $0.12 \mathrm{~mm}$.

Las diferencias fundamentales entre los valores obtenidos para las distintas vigas

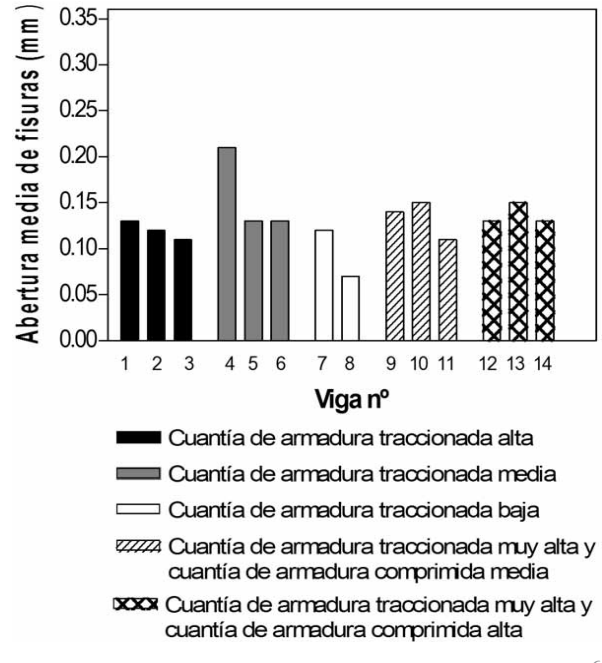

5. Valores experimentales de abertura media de fisuras principales $\left(\mathrm{w}_{\mathrm{p}}\right)$ para el escalón de servicio.

6. Valores de abertura media de fisuras principales $\left(w_{m}\right)$ según $E H E$ para el escalón de servicio.

Valores de abertura media de fisuras principales $\left(w_{m}\right)$ según EC-2 para el escalón de servicio. ensayadas residen en la distinta cuantía de armadura traccionada y en los diferentes diámetros empleados (a menor diámetro para una misma cuantía, menor abertura de fisura), y también se aprecia la influencia de la separación entre las armaduras longitudinales traccionadas.

En la Fig. 7 se muestran los valores de abertura media de fisuras para el escalón de servicio obtenidos de acuerdo con la formulación propuesta por EC-2. Esta formulación no tiene en cuenta la separación entre armaduras, aunque sí considera la influencia del diámetro, del recubrimiento y de la cuantía eficaz de armadura traccionada. Tampoco se considera la influencia de la disposición de armadura comprimida.

Para cuantías altas (incluyendo las vigas con y sin armadura comprimida) se obtienen valores comprendidos entre $0.09 \mathrm{~mm}$ y 0.12 $\mathrm{mm}$, para cuantías medias se obtienen valores entre $0.12 \mathrm{~mm}$ y $0.14 \mathrm{~mm}$, y para cuantías bajas se obtienen valores entre $0.12 \mathrm{~mm}$ y $0.16 \mathrm{~mm}$.

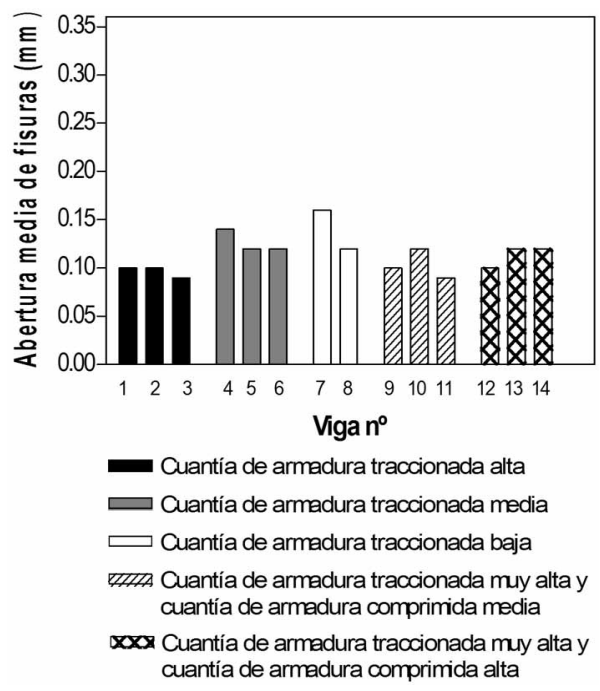


8. Valores de abertura media de fisuras principales $\left(w_{m}\right)$ según $\mathrm{ACl}$ para el escalón de servicio.

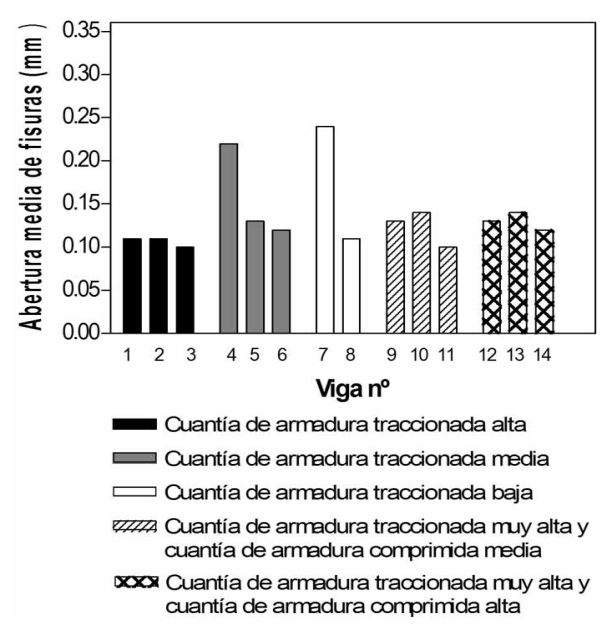

Las pequeñas variaciones que se observan entre las distintas vigas se deben fundamentalmente a los cambios de diámetro de las armaduras y a la variación de cuantía eficaz de armadura traccionada. De acuerdo con la formulación propuesta por EC-2, para mayor control de la fisuración se debe aumentar la cuantía eficaz de armadura traccionada, disponiendo diámetros finos.

En la Fig. 8 se muestran los valores de abertura media de fisuras para el escalón de servicio obtenidos de acuerdo con la formulación propuesta por Frosch (base del $\mathrm{ACl}$ ).

Como se mostró al exponer la formulación propuesta por Frosch, ésta no tiene en cuenta de forma directa el diámetro de las armaduras. Únicamente, se incluye dentro de la expresión del recubrimiento mecánico de las armaduras. Por lo tanto, en esta formulación no tiene gran influencia el cambio de diámetro. Tampoco se considera la influencia de la cuantía dispuesta ni la disposición de armadura comprimida.

Para cuantías altas (incluyendo las vigas con y sin armadura comprimida) se obtienen valores comprendidos entre $0.10 \mathrm{~mm}$ y 0.11 $\mathrm{mm}$, para cuantías medias se obtienen valores entre $0.12 \mathrm{~mm}$ y $0.22 \mathrm{~mm}$, y para cuantías bajas se obtienen valores entre $0.11 \mathrm{~mm}$ y $0.24 \mathrm{~mm}$.

Las diferencias fundamentales entre los valores obtenidos para las distintas vigas ensayadas residen en la separación entre las armaduras longitudinales traccionadas. En la gráfica de la Fig. 8 se puede observar cómo las vigas 4 y 7 presentan los mayores valores de abertura de fisura, al presentar la mayor separación entre armaduras.

Si se observan las gráficas de las Fig. 5 a 8, no se aprecia que ninguna de ellas tenga tendencias similares, ni respecto a los cam- bios de cuantía, ni respecto a los cambios de diámetro. Sí se observa una tendencia más similar entre las propuestas teóricas de EHE y EC-2, con ciertas diferencias que parecen estar asociadas a la consideración que hace EHE del parámetro separación de armaduras longitudinales traccionadas, no considerado por EC-2. Como se puede apreciar en la Fig. 8, dicho parámetro marca aún más la tendencia e crecimiento y/o decrecimiento de la abertura de fisuras según $\mathrm{ACl}$.

En la Fig. 9 se muestra una comparativa, en porcentajes, entre los valores de abertura media de fisuras para la carga de servicio propuestos por las formulaciones de las normativas consideradas, y los valores experimentales obtenidos.

Las formulaciones propuestas por EHE y ACI presentan una adecuación aceptable para cuantías altas y muy altas, con valores sensiblemente iguales o superiores a los experimentales. No obstante, para cuantías medias y bajas los valores propuestos por ambas normativas presentan una mayor dispersión respecto de los experimentales, con valores en general inferiores a éstos (relaciones de incluso el $40 \%$ y el $60 \%$ de la abertura de fisura experimental, para $\mathrm{EHE}$ y $\mathrm{ACl}$, respectivamente), salvo cuando la separación entre armaduras longitudinales es elevada. La formulación propuesta por EC-2 presenta cierta adecuación para cuantías altas y muy altas, si bien para cuantías medias y bajas en general resultan valores de abertura media de fisuras inferiores a los experimentales (relaciones de incluso el 50\% de la abertura de fisura experimental).

En la Fig. 10 mostramos un gráfico de la relación entre los valores de abertura media de fisuras propuestos por EHE y los valores experimentales, representando la línea de mejor ajuste entre ambos valores. Cabe señalar que en dicho gráfico ha sido omitido el valor correspondiente a la viga $\mathrm{n}^{\circ} 7$, dadas las incertidumbres de los resultados de dicho ensayo, al presentar la viga fisuras previas debidas a la contracción térmica inicial del hormigón. De dicho gráfico se deduce que la línea de mejor ajuste entre los valores experimentales y los teóricos, según EHE, se corresponde con una relación entre ambas de $\mathrm{W}_{\mathrm{EHE}}=0.99 \cdot \mathrm{W}_{\mathrm{EXP}}$. Dicha relación es indicativa de un buen ajuste medio, aunque con una dispersión relativamente importante. Este aspecto se observa en la Fig. 10 al quedar 5 de los 13 valores fuera de un intervalo de un $\pm 30 \%$ de ajuste respecto de la línea de ajuste ideal $\mathrm{w}_{\mathrm{EHE}}=\mathrm{w}_{\mathrm{EXP}}$.

En la Fig. 11 mostramos para EC-2 el mismo gráfico representado en la Fig. 10 para EHE. De dicho gráfico se deduce que la línea de 
mejor ajuste entre los valores experimentales y los teóricos según EC-2 se corresponde con una relación entre ambas de $\mathrm{w}_{\mathrm{EC}-2}=0.84 \cdot \mathrm{w}_{\mathrm{EXP}}$. Dicha relación es indicativa de un buen ajuste medio, aunque con valores propuestos menores a los experimentales. No obstante, aunque el ajuste medio es peor que el de EHE, la dispersión es menor según EC-2, al quedar sólo 1 valor de 13 fuera de un intervalo de un $\pm 30 \%$ de ajuste respecto de la línea de ajuste ideal $\mathrm{w}_{\mathrm{EC}-2}=\mathrm{w}_{\mathrm{EXP}}$.

De forma análoga, en la Fig. 12 mostramos el análisis realizado para $\mathrm{ACl}$, deduciendo una relación de $\mathrm{w}_{\mathrm{ACI}}=0.97 \cdot \mathrm{w}_{\mathrm{EXP}}$. $\mathrm{Al}$ igual que para $\mathrm{EHE}$, se obtiene un buen ajuste medio
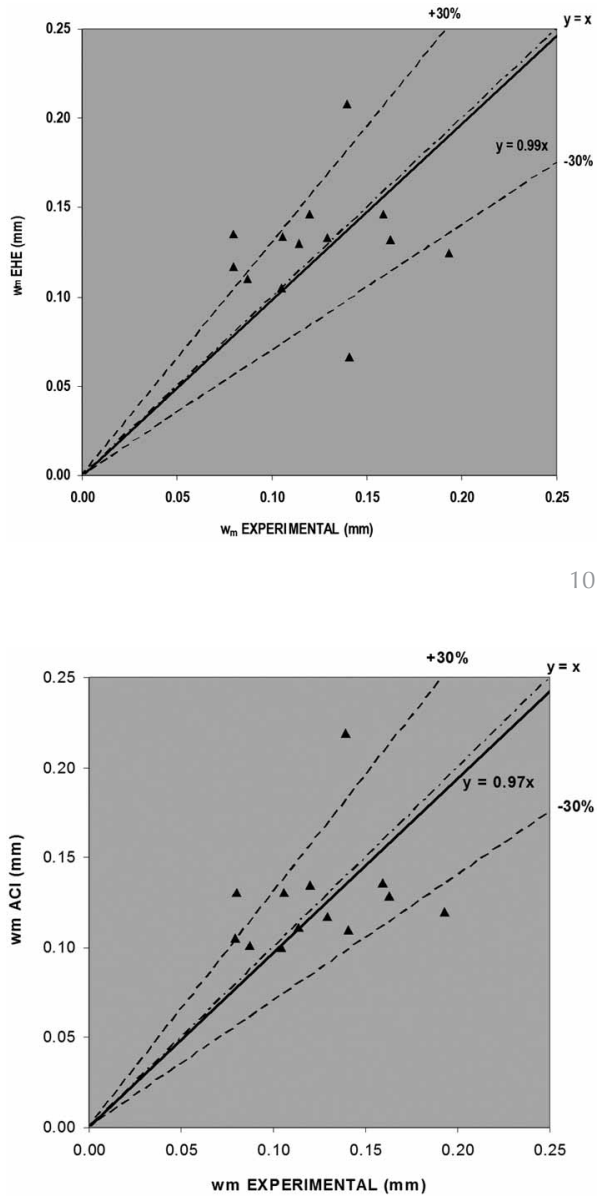

aunque con una dispersión relativamente importante.

Entre las formulaciones teóricas las que más se ajustan en términos medios a los resultados experimentales son las propuestas por EHE y ACl. No obstante, en los resultados experimentales no se observa la influencia tan acusada de la separación de armaduras que propone el $\mathrm{ACl}$, y en menor medida EHE. Además, como hemos indicado anteriormente, la dispersión del ajuste de valores propuestos por estas dos normativas es muy
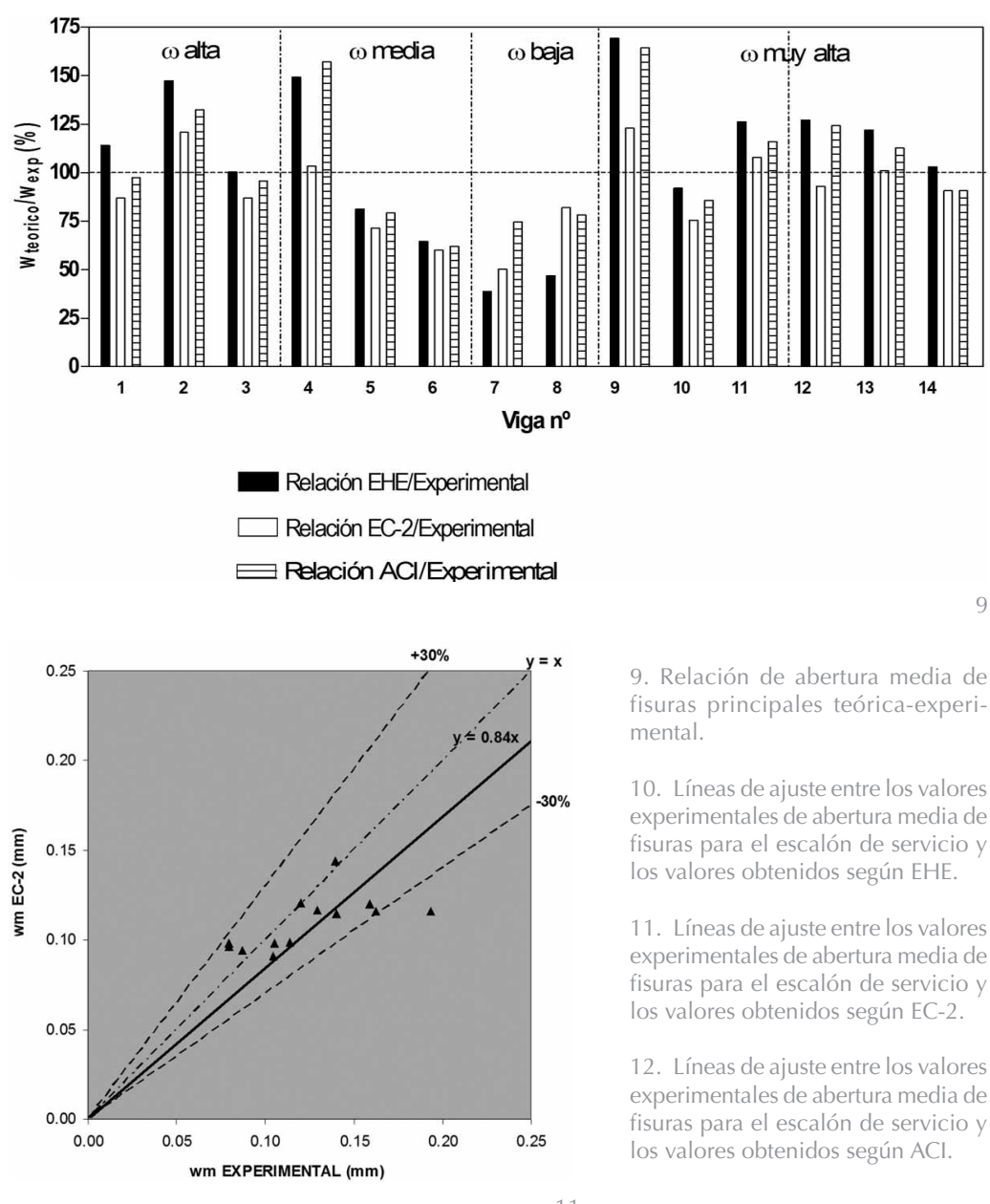

9. Relación de abertura media de fisuras principales teórica-experimental.

10. Líneas de ajuste entre los valores experimentales de abertura media de fisuras para el escalón de servicio y los valores obtenidos según EHE.

11. Líneas de ajuste entre los valores experimentales de abertura media de fisuras para el escalón de servicio y los valores obtenidos según EC-2.

12. Líneas de ajuste entre los valores experimentales de abertura media de fisuras para el escalón de servicio y los valores obtenidos según $\mathrm{ACl}$. elevada y superior a la de los valores propuestos por EC-2. En este sentido señalamos que la suma de residuos al cuadrado de los valores propuestos por $\mathrm{EHE}$ y $\mathrm{ACl}$ respecto de la línea de ajuste ideal $\left(\Sigma\left(\mathrm{w}_{\mathrm{EXP}}-\mathrm{w}_{\text {teórico: } \mathrm{EHE}}\right.\right.$ $\left.\left.{ }_{\mathrm{ACI}}\right)^{2}\right)$ es de 0.023 y 0.019 , respectivamente, valores superiores al obtenido para EC-2, que resulta 0.012. Además, si realizamos el análisis de residuos de los valores propuestos por las normativas respecto de la línea de ajuste obtenida para cada normativa, obtenemos que EC-2 presenta un valor de 0.006, muy inferior a los obtenidos para $\mathrm{EHE}$ y $\mathrm{ACl}$, 0.023 y 0.019 respectivamente. Este aspecto indica que pese a un peor ajuste medio de EC-2, los valores que propone reflejan mejor los datos experimentales que los propuestos por EHE y ACl.

\subsection{Separación de fisuras}

En la Fig. 13 se muestran los valores de separación media de fisuras obtenidos en los ensayos realizados para el escalón de servicio. En la gráfica se observa la influencia de la cuantía sobre la separación de fisuras: a mayor cuantía menor separación. También parece observarse una ligera influencia del diámetro dispuesto: a menor diámetro menor separación. 
13. Valores experimentales de separación media de fisuras principales $\left(\mathrm{s}_{\mathrm{m}}\right)$ para el escalón de servicio.

14. Valores de separación media de fisuras principales $\left(s_{m}\right)$ para el escalón de servicio según EHE.

15. Valores de separación media de fisuras principales $\left(\mathrm{s}_{\mathrm{m}}\right)$ para el escalón de servicio según EC-2.

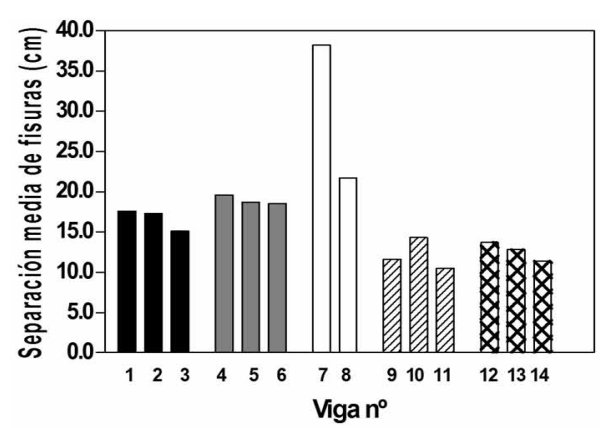

- Cuantía de armadura traccionada alta Cuantía de armadura traccionada media $\square$ Cuantía de amadura traccionada baja VIII Cuantía de amadura traccionada muyalta ycuantía de amadura comprimida media

XXuantía de armadura traccionada muyalta ycuantía de armadura comprimida alta
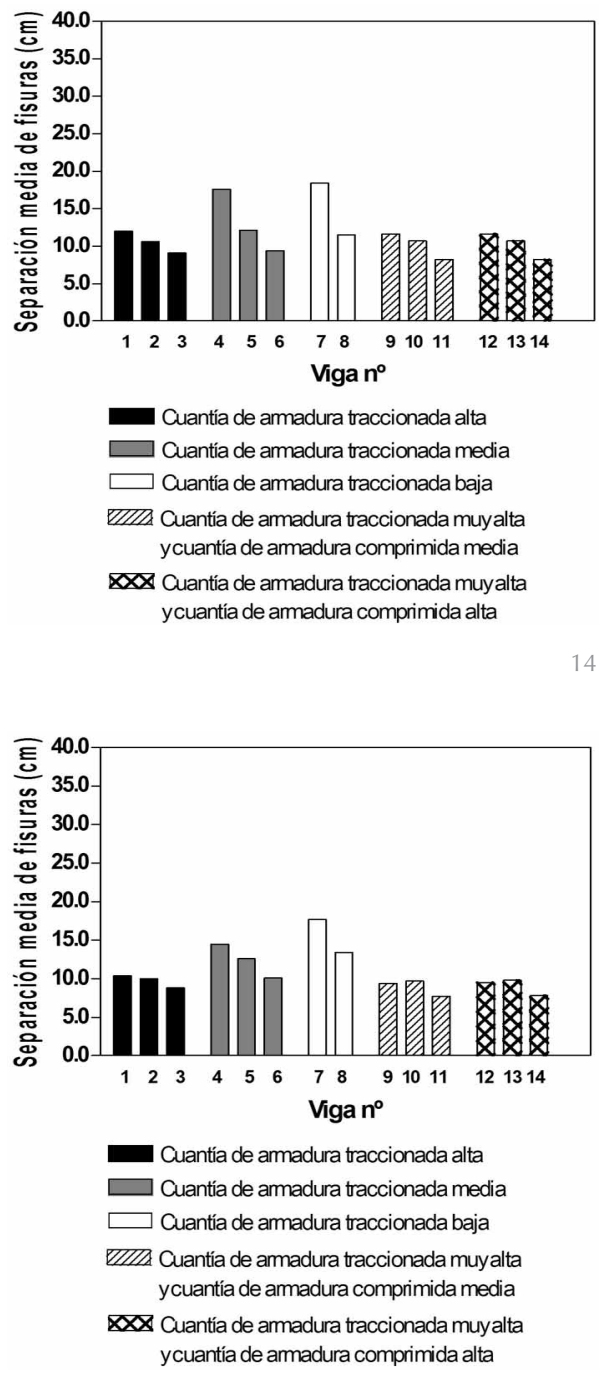

Para cuantías altas se obtienen valores entre $15.1 \mathrm{~cm}$ y $17.6 \mathrm{~cm}$, para cuantías medias se obtienen valores entre $18.5 \mathrm{~cm}$ y $19.6 \mathrm{~cm}$, y para cuantías bajas se obtienen valores entre $21.7 \mathrm{~cm}$ y $38.2 \mathrm{~cm}$. Cabe señalar que en el caso de la viga 7, la fisuración previa parece haber condicionado la posición de las fisuras aparecidas durante el ensayo.

En cuanto a las vigas de cuantía elevada, con y sin armadura comprimida, no se observa influencia alguna en la disposición de la misma. Los resultados obtenidos están comprendidos entre $10.5 \mathrm{~cm}$ y $14.3 \mathrm{~cm}$, es decir, menor separación de fisuras que las vigas de cuantía alta (vigas 1 a 3), al ser la cuantía aún mayor.

En la Fig. 14 se muestran los valores de separación media de fisuras para el escalón de servicio obtenidos de acuerdo con la formulación propuesta por EHE. Tal y como se indica en la formulación de EHE a mayor cuantía de armadura traccionada y menor diámetro de éstas menor separación de fisuras. Asimismo, también se aprecia la influencia que otorga la formulación a la separación de armaduras traccionadas, deduciéndose que las vigas que mayor separación presentan (vigas 4 y 8) son para las que mayor separación media de fisuras se obtiene (de unos $18 \mathrm{~cm}$ ). Para cuantías altas, diámetros reducidos y separación de armaduras traccionadas mínima, se deduce una separación media de fisuras de $8.2 \mathrm{~cm}$ (vigas 11 y 14). Como se ha indicado para la abertura media de fisuras, esta formulación no considera la influencia de la disposición de armadura comprimida.

En la Fig. 15 se muestran los valores de separación media de fisuras para el escalón de servicio obtenidos de acuerdo con la formulación propuesta por EC-2. En la fórmula propuesta la mayor influencia la tiene el diámetro, el recubrimiento y de la cuantía eficaz de armadura traccionada. No se considera la influencia de la disposición de armadura comprimida.

Para cuantías altas (incluyendo las vigas con y sin armadura comprimida) se obtienen valores comprendidos entre $7.7 \mathrm{~cm}$ y $10.4 \mathrm{~cm}$, para cuantías medias se obtienen valores entre $10.1 \mathrm{~cm}$ y $14.5 \mathrm{~cm}$, y para cuantías bajas se obtienen valores entre $13.4 \mathrm{~cm}$ y $17.7 \mathrm{~cm}$.

Como se deduce de la formulación del EC-2 y de la gráfica de la Fig. 15 a mayor cuantía de armadura dispuesta menor es la separación media de fisuras. Las desviaciones dentro de cada cuantía se deben a la modificación del diámetro, a menor diámetro menor separación de fisuras.

En la Fig. 16 se muestran los valores de separación media de fisuras para el escalón de servicio obtenidos de acuerdo con la formulación propuesta por Frosch (base del ACI). Como ya se indicó anteriormente, dicha formulación está fundamentalmente condicionada por el 
recubrimiento mecánico de las armaduras y por la separación entre éstas. Para las vigas ensayadas, al ser los recubrimientos similares, en los resultados de la Fig. 16 ha resultado condicionante la separación entre armaduras. Tampoco se considera la influencia de la cuantía dispuesta ni la disposición de armadura comprimida.

Para una separación de armaduras reducida (vigas 3, 6, 8, 11 y 14), entre $3 \mathrm{~cm}$ y $4 \mathrm{~cm}$, los valores de separación media de fisuras deducidos están entre $7.0 \mathrm{~cm}$ y $7.7 \mathrm{~cm}$. Para una separación de armaduras elevada (vigas 4 y 7), de unos $16 \mathrm{~cm}$, los valores de separación media de fisuras deducidos son de unos $16 \mathrm{~cm}$.

Si se observan las gráficas de las Fig. 13 a 16 , se aprecia que los valores experimentales obtenidos de separación media de fisuras son del orden del doble de los deducidos según las normativas elegidas para la comparación. Las gráficas tienen tendencias similares, reduciéndose siempre la separación de fisuras para cada grupo de cuantía. En la Fig. 13 se observa que en los valores experimentales obtenidos sí se aprecia cierta influencia de la cuantía dispuesta al igual que los valores propuestos por el EHE y EC-2. No obstante, en la Fig. 13 no se observa la influencia de la separación de armaduras longitudinales que se aprecia en la Fig. 16 para $\mathrm{ACl}$, y en menor medida para EHE (véase la Fig. 14).

En la Fig. 17 se muestra una comparativa, en porcentajes, entre los valores de separación media de fisuras para la carga de servicio propuestos por las formulaciones consideradas, y los valores experimentales obtenidos.

De esta gráfica se deduce que la separación media de fisuras propuesta por las tres normativas consideradas es siempre muy inferior a la obtenida experimentalmente, sobre todo para cuantías bajas, del orden del 50\% (y hasta del $35 \%$ en el caso del $\mathrm{ACl}$ ), salvo para cuantías muy elevadas en las que es un $25 \%$ inferior.

Al igual que se expuso para la abertura media de fisuras, en las Figs. 18 a 20 mostramos unos gráficos de la relación entre los valores de separación media de fisuras propuestos por EHE, EC-2 y ACl, y los valores experimentales, representando las líneas de mejor ajuste entre valores teóricos y experimentales. Al igual que en el caso de la abertura media de fisuras ha sido omitido el valor correspondiente a la viga $n^{\circ} 7$. De dichos gráficos se deduce que las líneas de mejor ajuste entre los valores experimentales y los teóricos resultan en general muy inferiores (entre un 30\% y un $45 \%$ ) a las correspondiente al ajuste ideal. Los valores propuestos por $\mathrm{EHE}$ y $\mathrm{ACI}$ presentan

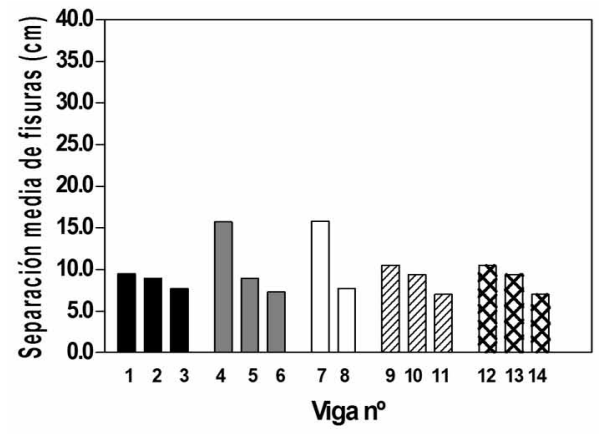

16. Valores de separación media de fisuras principales (s ) para el escalón de servicio según $\mathrm{ACl}$.

7. Relación de separación media de fisuras principales teóricaexperimental.

18. Líneas de ajuste entre los valores experimentales de separación media de fisuras para el escalón de servicio y los valores obtenidos según EHE.
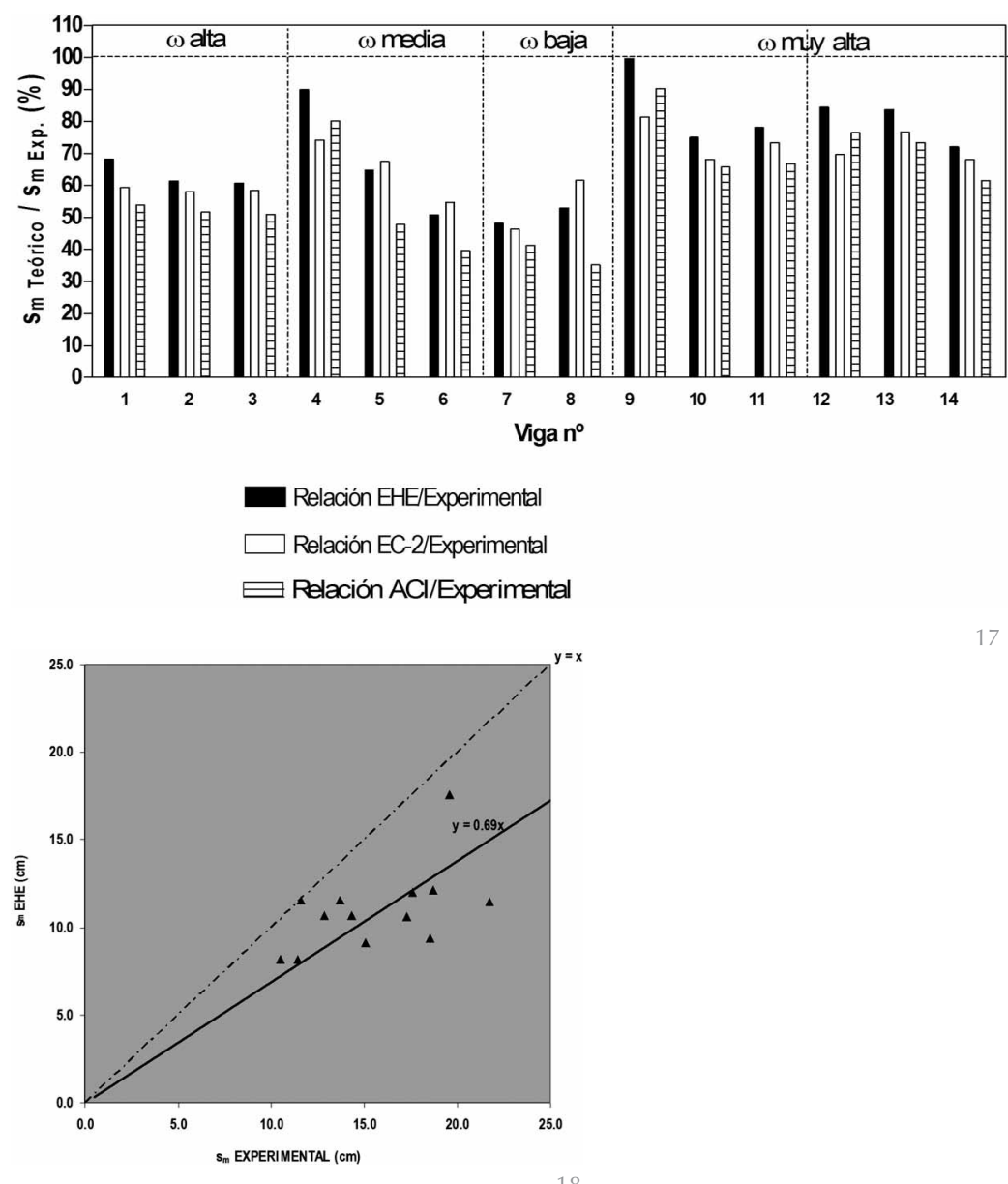

una dispersión notable respecto la línea de mejor ajuste, si bien los valores propuestos por EC-2 presentan una dispersión aceptable respecto su línea de mejor ajuste.

Por lo tanto, se deduce que aunque los valores propuestos por las tres normativas empleadas son siempre muy inferiores a los valores experimentales obtenidos, la formulación que 
19. Líneas de ajuste entre los valores experimentales de separación media de fisuras para el escalón de servicio y los valores obtenidos según EC-2.

20. Líneas de ajuste entre los valores experimentales de separación media de fisuras para el escalón de servicio y los valores obtenidos según $\mathrm{ACl}$.
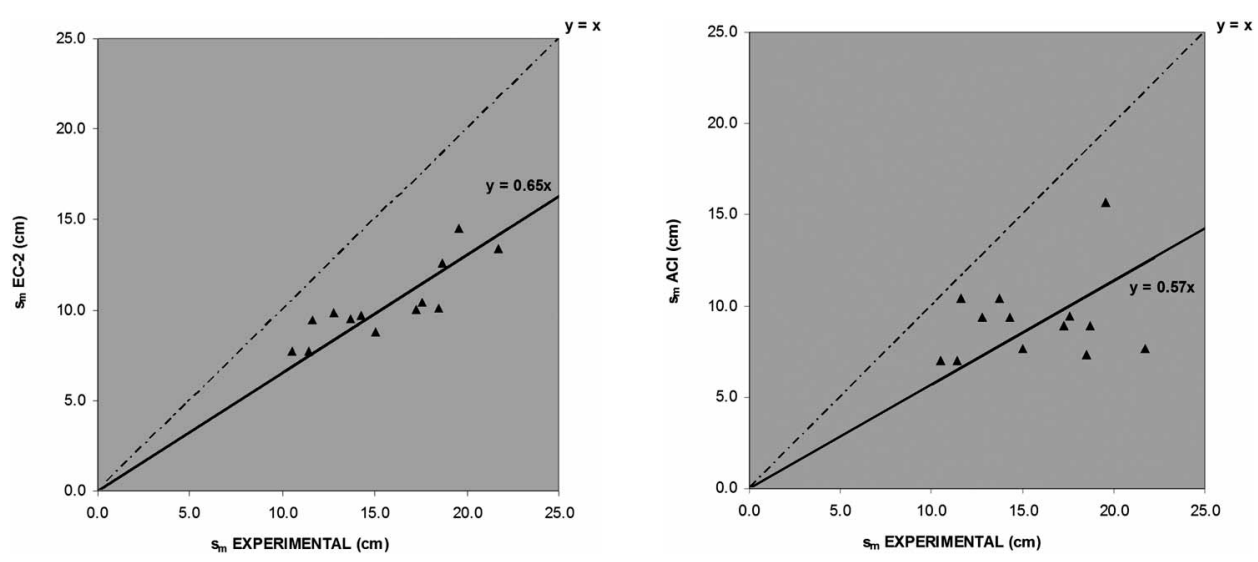

en general mejor se ajusta a la situación real, es la propuesta por el EC-2.

\section{PROPUESTA DE MEJORA DE LAS FORMULACIONES PARA LA OBTENCIÓN DE SEPARACIÓN MEDIA Y ABERTURA MEDIA DE FISURAS}

Con base en los resultados obtenidos en este trabajo de investigación, en el presente apartado se han tratado de plantear con carácter preliminar las posibles variantes de las fórmulas existentes, con objeto de mejorar el ajuste de las mismas para la obtención de la separación media y abertura media de fisuras.

En primer lugar, se ha establecido como punto de partida la formulación propuesta por EC-2, puesto que de acuerdo con los resultados expuestos en la presente investigación, es la formulación que plantea un ajuste más aproximado (aunque minusvalorando los valores obtenidos) a los resultados experimentales.

En cuanto a la separación media de fisuras se han planteado tres posibles propuestas. Para la definición de dichas propuestas se ha tenido en cuenta una de las conclusiones obtenidas en la presente investigación: la falta de influencia significativa del diámetro de las armaduras en el control de la fisuración. Por lo tanto, como se muestra más adelante, las tres propuestas se diferencian en la consideración del parámetro diámetro. Para cada una de las propuestas se han realizado iteraciones sucesivas hasta obtener las constantes que proporcionaban tanto el ajuste promedio ideal de resultados respecto los valores experimentales y la menor dispersión de los mismos respecto la línea de mejor ajuste de la fórmula propuesta.

De acuerdo con dicho análisis se han obtenido las siguientes expresiones $\left(\mathrm{s}_{\mathrm{m}^{\prime}} \mathrm{c}\right.$ y $\phi$ en $\mathrm{mm}$ ):
Propuesta 1:

$\mathrm{s}_{\mathrm{m}}=3.10 \cdot \mathrm{c}+0.15 \cdot \frac{\phi}{\rho_{p, \text { eff }}}$

Propuesta 2:

$\mathrm{s}_{\mathrm{m}}=2.96 \cdot \mathrm{c}+0.65 \cdot \frac{\sqrt{\phi}}{\rho_{p, \text { eff }}}$

Propuesta 3:

$\mathrm{s}_{\mathrm{m}}=3.09 \cdot \mathrm{c}+2.26 \cdot \frac{1}{\rho_{p, \text { eff }}}$

Del análisis de las líneas de ajuste de dichas propuestas respecto a los resultados experimentales y concretamente del análisis de residuos al cuadrado de estas propuestas, la propuesta 2 es la que menor dispersión de resultados presenta para un mismo ajuste promedio de la fórmula planteada.

Una vez planteadas las propuestas para la en cuanto a la abertura media de fisuras se pueden establecer dos distintas consideraciones del valor de deformación media del acero: la consideración del efecto de tensionstiffening de forma análoga a como lo adopta EC-2, según la expresión que anteriormente fue indicada para la estimación de la deformación media del acero $\left(\varepsilon_{\mathrm{m}}\right)$; adoptar como para la sección fisurada $\left(\varepsilon_{\mathrm{s} 2}\right)$.

Una vez más, se deducen los coeficientes que deben multiplicar a la deformación media del acero considerada para obtener el ajuste ideal de cada una de las propuestas. Dichos coeficientes resultan ser prácticamente idénticos para ambas propuestas (en cuanto la separación media de fisuras), por lo que resulta:

$\mathrm{W}_{\mathrm{m}}=\mathrm{S}_{m} \cdot\left(0.78 \cdot \varepsilon_{m}\right)$

Consideración efecto tension-stiffening

$\mathrm{W}_{\mathrm{m}}=\mathrm{S}_{m} \cdot\left(0.58 \cdot \varepsilon_{\mathrm{s} 2}\right)$

No consideración efecto tension-stiffening estimación de la separación media de fisuras, deformación media del acero la producida 
Como se deduce del análisis de residuos al cuadrado, la propuesta 2 (en cuanto la separación media de fisuras) es la que menor dispersión de resultados presenta para un mismo ajuste promedio de la fórmula planteada, tanto para la consideración del efecto de tension-stiffening que propone EC-2 como la simplificación de adoptar como deformación media la deformación del acero en las secciones fisuradas.

Por todo lo anteriormente expuesto se pueden establecer como propuestas para la estimación de abertura media de fisuras las siguientes:

Propuesta 2.1:

$\mathrm{W}_{\mathrm{m}}=\left(2.96 \cdot c+0.65 \cdot \frac{\sqrt{\phi}}{\rho_{p, e f f}}\right) \cdot\left(0.77 \cdot \varepsilon_{m}\right)[20]$

$\varepsilon_{\mathrm{m}}$ según fórmula EC-2

Propuesta 2.2:

$\mathrm{W}_{\mathrm{m}}=\left(2.96 \cdot c+0.65 \cdot \frac{\sqrt{\phi}}{\rho_{p, e f f}}\right) \cdot\left(0.58 \cdot \varepsilon_{s 2}\right)[21]$

$\varepsilon_{\mathrm{s} 2}$ (estado fisurado)

De estas propuestas la que menor dispersión presenta para el mejor ajuste promedio es la correspondiente a la propuesta 2.1. No obstante, la falta de sentido físico de multiplicar la deformación media del acero propuesta por EC-2 por un coeficiente, en lugar de corregir la propia fórmula que dicha normativa plantea para la estimación de la deformación media, hace que resulte más adecuado y simplificado adoptar la fórmula de la propuesta 2.2 en la que simplemente se reduce la deformación del acero para la sección fisurada (de fácil estimación). De hecho, el coeficiente que se propone $(0.58)$ es muy próximo al umbral inferior que propone EC-2 para la deformación media del acero $\left(0.6 \cdot \varepsilon_{\mathrm{s} 2}\right)$.

Por lo tanto, se puede establecer como propuesta de mejora de la estimación de abertura media de fisuras la siguiente:

$\mathrm{W}_{\mathrm{m}}=\left(3 \cdot c+0.65 \cdot \frac{\sqrt{\phi}}{\rho_{p, e f f}}\right) \cdot\left(0.58 \cdot \varepsilon_{s 2}\right)$

\section{CONCLUSIONES}

Del análisis de resultados obtenidos en la investigación experimental realizada, establecemos las siguientes conclusiones:
1. Se observa un buen ajuste medio entre los resultados experimentales obtenidos y los propuestos por las normativas EHE, $\mathrm{ACl} 318$ Building Code y Eurocódigo 2 para la abertura media de fisuras en estructuras de hormigón sometidas a esfuerzos de flexión pura. Para cuantías bajas y medias los resultados experimentales son superiores a los teóricos, si bien para cuantías altas son sensiblemente iguales o incluso en ocasiones inferiores. En el caso de los valores propuestos por $\mathrm{EHE}$ y $\mathrm{ACl}$, el ajuste promedio es muy bueno, aunque con mayor dispersión que en el caso de EC-2. Esta normativa ofrece un ajuste medio peor que las anteriores, ya que minusvalora la abertura media de fisuras, pero su dispersión es mucho menor, y ofrece resultados que reflejan mejor los valores experimentales. Además, aunque la formulación propuesta por el $\mathrm{ACl}$ presenta una mejor adecuación en términos globales, en algunos casos dicha normativa es muy conservadora, al penalizar en gran medida las vigas que presentan un valor de separación entre armaduras longitudinales elevado, influencia que no se percibe en los resultados experimentales obtenidos. Dicho aspecto se da en menor medida para EHE, que también tiene en cuenta la separación entre armaduras longitudinales.

2. Los valores de separación media de fisuras experimentales son, aproximadamente, entre 1.5 y 2 veces los deducidos según las normativas antes referidas, con una ligera mejor adecuación del EC-2 respecto de EHE y ACl. $\mathrm{Al}$ igual que para la abertura media de fisuras, se da una mejor adecuación para cuantías elevadas. También se observa una mayor dispersión de resultados entre los valores propuestos por EHE y ACl, y los valores propuestos por EC-2, en algunos casos, debidos fundamentalmente a la importancia que da $\mathrm{ACl}$, y en menor medida EHE, a la separación de armaduras longitudinales, parámetro que no parece tener influencia en los resultados experimentales obtenidos.

3. De los resultados experimentales obtenidos se deduce que la cuantía mecánica tiene influencia en el control de la fisuración. A mayor cuantía dispuesta menor separación y abertura de fisuras, y menos necesaria se hace la comprobación de la fisuración en el estado límite de servicio tras haber diseñado la pieza por condiciones de seguridad. Dicho parámetro es parte fundamental de la formulación propuesta por EC-2.

4. De los resultados experimentales obtenidos se deduce que el parámetro diámetro está sobrevalorado por las normativas consideradas en el control de la fisuración. En el caso de la separación media de fisuras se observa una ligera influencia, pero ésta no parece ser tan significativa como indican EHE o EC-2. 
5. No existe influencia alguna de la disposición de armadura comprimida en el control de la fisuración, influencia que tampoco es contemplada por las normativas consideradas.

6. Como posible mejora de la formulación de EC-2, que parece ser un modelo más ajustado a los valores obtenidos por su poca dispersión, se propone la siguiente:

$\mathrm{W}_{m}=\mathrm{s}_{m} \varepsilon_{m}$

$\mathrm{W}_{\mathrm{m}}=\left(3 \cdot c+0.65 \cdot \frac{\sqrt{\phi}}{\rho_{p, e f f}}\right) \cdot\left(0.58 \cdot \varepsilon_{\mathrm{s} 2}\right)$
Siendo c el recubrimiento geométrico en $\mathrm{mm}$, $\phi$ el diámetro de las armaduras en $\mathrm{mm}, \rho_{p \text {, eff }}$ la cuantía eficaz según EC-2 y $\varepsilon_{s 2}$ la de formación del acero para las secciones fisuradas. Con ello se reduce el efecto del diámetro y se simplifica el cálculo de la deformación media del acero.

\section{AGRADECIMIENTOS}

Los autores quieren expresar su más sincero agradecimiento al Instituto Técnico de Materiales y Construcciones (INTEMAC), por financiar esta investigación, así como otras tantas líneas de investigación relacionadas con el comportamiento de las estructuras de hormigón y el desarrollo de la construcción en general.

\section{BIBLIOGRAFÍA}

(1) Schiessl, P., "Admissible crack width in reinforced concrete structures", Preliminary Reports Tome /I of IABSEFIP-CEB-RILEM-IASSS-Coloquium, Liège, June 1975.

(2) Darwin, D. et al., "Debate: Crack Width, Cover, and Corrosion", Concrete International, V. 8, No 5, May 1985, pp. 20-35.

(3) Perepérez, B.; Barbera, E.; and Benlloch, J., "Grandeurs de fissures et corrosion". Conférence Européenne «La Fissuration des Bètons et la Durabilité des Constructions», AFREM-CEE, 1988.

(4) Andrade, C.; Molina, F.J.; and Alonso, C., "Cover Cracking as a Function of Rebar Corrosion: Part I-Experiment Test", Materials and Structures, V. 26, 1993, pp 453-464.

(5) Alonso, C.; Andrade, C.; Rodríguez, J.; and Díez, J.M., "Factors Controlling Cracking of Concrete Affected by Reinforcement Corrosion", Materials and Structures, V. 31, No. 8, 1998, pp. 435-441.

(6) Campbell-Allen, D., "The reduction of cracking in concrete", University of Sidney, May 1979.

(7) Padilla, J.D.; and Robles, F., "Human response to cracking in concrete slabs". ACI Special Publications SP-30 "Cracking. Deflection and Ultimate Load of Concrete Slab Systems", American Concrete Institute, Detroit, 1971.

(8) Broms, B., "Crack Width and Crack Spacing in Reinforced Concrete Members", ACl Journal, Proceedings V. 62, No 10, Oct 1965, pp. 1237-1255.

(9) Gergely, P.; and Lutz, L.A., "Maximum Crack Width in Reinforced Concrete Flexural Members", Causes, Mechanism, and Control of Cracking in Concrete, SP-20, American Concrete Institute, Farmington Hills, Mich., 1968, pp. $87-117$.

(10) Ferry-Borges, J., "Cracking and deformability of reinforced concrete beams", Association International des Ponts et Charpenters, 26, 1966.

(11) Base, G.D.; Read, J.B.; Beeby, A.W.; Taylor, H.P.J. "An investigation of the crack control characteristics of various types of bar in reinforced concrete beams", Cement and Concrete Association, London, 1966.

(12) Beeby, A.W. "An Investigation of Cracking in Slabs Spanning One Way", Cement and Concrete Association, Technical Report No. TRA 433, London, April 1970.

(13) Nawy, E.G., "Crack Control in Reinforced Concrete Structures", ACl Structural Journal, V. 65, No. 10, Oct. 1968.

(14) Frosch, R.J. "Another Look at Cracking and Crack Control in Reinforced Concrete", ACl Structural Journal, V.96, No 3, May-June 1999

(15) Frosch, R.J. "Modeling and Control of Side Face Beam Cracking", ACl Structural Journal, V. 99, No 3, May-June 2002.

(16) Instrucción de Hormigón Estructural 2008. EHE. Madrid (España), 18 de julio de 2008.

(17) Eurocode 2. "Design of Concrete Structures". Part 1-1: General rules and rules for buildings, Brussels, Belgium, 2004.

(18) ACl Committee 318, "Building Code Requirements for Structural Concrete (ACl 318M-08) and Commentary", American Concrete Institute, Farmington Hills, Mich., 2008.

(19) ACl 318-95. "American Concrete Institute. Building Code Requirement for Reinforced Concrete (ACl 318-95) and Commentary (ACI 318R-95)", American Concrete Institute, Farmington Hills, Mich., 1995.

(20) ACl Comittee 318, "Proposed Revisions to Building Code Requirements for Structural Concrete (ACl 318-95) and Commentary (ACl 318R-95)", Concrete International, May 1999.

(21) ACl 318-99. "American Concrete Institute. Building Code Requirement for Reinforced Concrete (318-99) and Commentary (ACl 318R-99)", American Concrete Institute, Farmington Hills, Mich., 1999.

(22) ACl Committee 318, "Building Code Requirements for Structural Concrete (ACl 318-02) and Commentary (318R06)", American Concrete Institute, Farmington Hills, Mich., 2006.

(23) Beeby, A.W., "The influence of the parameter $\phi / \rho_{\text {eff }}$ on crack widths", Structural Concrete, V. 5, No 2, 2004.

(24) Nejadi, S.; and Gilbert, I., "Shrinkage Cracking and Crack Control in Restrained Reinforced Concrete Members", ACl Structural Journal, V. 101, No 6, November-December 2004.

(25) Peralta, M.H.; Rivas, I.E.; Ortega, N.F., "Análisis numérico de la fisuración superficial de estructuras de hormigón armado por efecto de la corrosión", Informes de la Construcción, Vol. 58, No 501, 2006.

$$
* * *
$$

